\title{
Complications of Hysteroscopic Surgery
}

\author{
Surg Capt (Mrs) P Tarneja ${ }^{*}$, Surg Capt VK Tarneja ${ }^{+}$, Lt Col BS Duggal
}

MJAFT 2002; 58 : 331-334

Key Words : Complications; Correct surgical techniques; Distension media; Preventable

\section{Introduction}

D iagnostic and operative hysteroscopy are safe procedures and many large series of operative procedures are reported with no complications. As hysteroscopy continues to proliferate as a diagnostic and therapeutic tool for intrauterine abnormalities and is becoming extremely popular amongst gynaecologists, the importance of preventing, recognizing and appropriately managing hysteroscopic complications rises. Complications tend to occur mostly when contraindications are ignored and when incorrect surgical techniques or instruments are used. Some potential problems are inherent in therapeutic or operative hysteroscopic procedures and are more frequent with diagnostic hysteroscopy.

\section{Complications of Distension Media}

Panoramic hysteroscopy requires uterine distension for diagnostic and operative procedures. There are three basic distending media : high molecular weight dextran, carbon dioxide $\left(\mathrm{CO}_{2}\right)$ and low viscosity fluids. Complications do not result from mere presence of medium in the uterine cavity but because medium enters the vascular system. Some vascular intravasation is inherent in all hysteroscopic procedures $[1,2]$.

The factors that influence the amount of intravasation are the pressure used to distend the uterine cavity, the type of operation and the duration of procedure. Difficult dilatation causing laceration, hysteroscopic myomectomy and resection of endometrium, can open vascular channels and increase intravasation. Whenever fluids are used, the input and outflow of distending media must be monitored carefully.

$\mathrm{CO}_{2}$ is mainly used for diagnostic hysteroscopy because it does not allow clearing of debris from uterine cavity during operative procedures. When used at a maximal flow rate of $100 \mathrm{ml} / \mathrm{m}$ and pressure of $100 \mathrm{mmHg}$, controlled with Hysteroflator, $\mathrm{CO}_{2}$ is a safe medium. Although embolisation can occur with controlled pressure, it is not dangerous as $\mathrm{CO}_{2}$ is dissolved readily in blood and released during ventilation
[3]. With uncontrolled pressures there can be increased $\mathrm{PCO}_{2}$ resulting in metabolic acidosis and cardiac irregularities.

Dextran 70 (Hyskon) possesses high viscosity that renders it immiscible with blood which allows clear visualisation in presence of bleeding. However, problems related with Hyskon are pulmonary oedema, allergic reactions and coagulopathies. Hyskon being highly viscous, high pressures are required to create adequate flow into uterine cavity. Due to high pressure, more dextran gets intravasated causing expansion of plasma volume and pulmonary oedema $[4,5]$. Dextran is also known to have anti clotting properties [6]. Ellingson and Aboulafia [7] described four problems associated with dextran - hypotension, hypoxia, coagulopathy and anaemia and named it Dextran syndrome. Minor allergic reactions and anaphylaxis to dextran are reported. Hapten inhibitors are available to prevent this problem but are not used because serious allergic reactions are uncommon, $1: 1500$ to $1: 300,000$. Low viscosity fluids are the most commonly used distension media. The major complications of low viscosity media result from excessive absorption and consequent fluid overload. Low viscosity media can be divided in two groups depending on their tonicity and electrolyte content : hypotonic electrolyte free media and isotonic electrolyte containing media.

The hypotonic group includes glycine and sorbitol. These solutions being electrolyte free are nonconductive, hence preferred during electrosurgical procedures. Being hypotonic and electrolyte free, excessive absorption of this fluid causes hypervolaemia and hyponatraemia. After intravasation, glycine and sorbitol get metabolized leaving free water in intravascular space which moves by osmosis into intracellular and extracellular space. Free water accumulates in brain tissue which increases pressure and causes cellular necrosis. Clinically it manifests as nausea, vomiting, agitation and headache. If untreated it may progress to bradycardia and hypertension and subsequently hypotension, pulmonary oedema, cerebral oedema and car-

\footnotetext{
"Professor and Head, Department of Obstetrics and Gynaecology, ${ }^{+}$Professor and Head, Department of Anaesthesiology and Critical Care, Armed Forces Medical College, Pune - $411040,{ }^{*}$ Classified Specialist (Obstetrics and Gynaecology), Military Hospital, Shillong - 793007.
} 
diovascular collapse [8]. Premenopausal women are 25 times more prone to develop this complication because the cation pump of cerebral neurons which reduces cerebral oedema by throwing out osmotically active cations, remains inhibited by sex hormones [9]. Glycine is metabolized to ammonia which at higher concentrations contributes to muscle aches, visual disturbances and encephalopathy.

In our series of 85 hysteroscopic procedures in cases of infertility and 60 TCRE we had one case of excessive glycine absorption after TCRE. Patient had blurring of vision for one day postoperatively. In addition patient had low sodium and responded well to saline infusion. Recently mannitol has been evaluated as distension media. It is electrolyte free and also isotonic. It does not get metabolized to ammonia so there are less chances of encephalopathy [10].

The isotonic electrolyte containing distension media commonly used are normal saline and Ringer's lactate. Their sodium content and their osmolality prevent hyponatraemic encephalopathy. Since these media are used only for diagnostic hysteroscopy, fluid overload is not seen with these media. We have used these media for more than thousand diagnostic hysteroscopies and found them absolutely safe. In case these media can be used for operative hysteroscopy, it will dramatically decrease the risk of hyponatraemia and hypo-osmolality [11]. Versapoint is an example of bipolar operating system that conducts electric current between two electrodes that are in close proximity and hence can be used with electrolyte containing isotonic media. Versapoint is useful for small polyps and synechiae but is not suitable for resecting large myomas or endometrial ablation. ERA sleeve and OPERA star system are modifications of unipolar system where retum electrode is in close proximity to active electrode rather than on patient's thigh. Higher power settings are required with this system which can lead to formation of bubbles and potential danger of gas emboli.

Problem of fluid overload is one of those complications of hysteroscopic surgery which can be easily prevented by following certain guidelines. Excessive infusion pressure that results in excessive intrauterine pressure is the most important risk factor. We have used Endomat for most of our cases but whenever we have used pressure bag, we followed the basic principle to use lowest pressure to achieve clear view of uterine cavity. In our experience, pressure reflected by manometer is not the true intrauterine pressure.

Operative procedures that last more than one hour and incorporate resection of large amounts of tissue are more likely to lead to fluid overload complica- tions. Maximum operating time in our cases was $\mathbf{5 0}$ minutes. Strictly following this principle we resected sub mucus myomas in two sittings in two cases.

Traumatic cervical dilatation and uterine perforation create vascular rents and increase fluid absorption. Expanding hydrophilic dilators can be used preoperatively in post menopausal women with stenosed cervix. In one case, while doing TCRE, perforation took place but procedure could be completed. Immediately after the procedure we realized fluid deficit was more than 2 litres. During laparoscopic evaluation for perforation, 1.5 litres of fluid was removed from peritoneal cavity. Patient made an uneventful recovery but it is always advisable to abandon the procedure as soon as perforation takes place.

Use of vasoconstrictor agents like vasopressin has been associated with less intravasation but is not recommended because of its antidiuretic effect. Preoperative use of gonadotropin releasing hormone agonists has the advantage of causing less intravasation but at the same time softens the myometrium and increases the risk of perforation.

Meticulous measurement of inflow and outflow of distension media is the most important factor to prevent fluid overload. Automated fluid management systems that continuously display distension media deficit are ideal, however, we keep one operating room staff to monitor and alert the surgeon and anaesthesiologist as soon as deficit exceeds $1000 \mathrm{ml}$. If deficit exceeds $1500 \mathrm{ml}$ it is advisable to terminate the procedure.

\section{Uterine Perforation}

Uterine perforation is the commonest complication of hysteroscopic surgery. Uterus can be perforated during dilation or with hysteroscope. Cervical stenosis, severe uterine anteflexion or retroflexion, synechiae, myoma resection, endometrial resection, septa division and operator inexperience, all increase the risk of perforation.

\section{Infection}

Infection is an uncommon complication of operative hysteroscopy. Various risk factors are : history of pelvic inflammatory disease, pre-operative use of laminaria tent, long operative procedure, repeated insertion and removal of hysteroscope through cervix and tissue fragments left in utero. Most post operative infections are cystitis, endometritis, pyometra, and rarely parametritis, tubo-ovarian abscess and broad ligament abscess. Although prophylactic antibiotics have not been demonstrated to reduce the incidence of postoperative infection; we have used broad spectrum antibiotics for all operative hysteroscopies and had no post operative infections. 


\section{Electrosurgical Complications}

The important intraoperative or postoperative complications from electrosurgical devices are thermal injuries to viscera which can lead to peritonitis, sepsis and death. Thermal injuries usually follow perforation, however, thermal bowel injury in absence of uterine perforation has been reported [14]. Electrosurgical thermal effects with monopolar current are complex and not fully understood Undesired energy transfer from active electrode to viscera can occur due to faulty insulation, direct coupling and capacitative coupling.

Proper instrumentation and activation of electrode always under vision, will prevent this undesirable energy transfer but jumping of electrical energy due to capacitative coupling is beyond the control of surgeon. Fortunately, capacitative coupling is not reported with hysteroscopic surgery. We had no electro-surgical complications as we always followed the basic principle of keeping active electrode under vision.

\section{Air Embolism}

Air embolism is a rare but most dangerous and potentially fatal complication of hysteroscopic surgery. Brooks has reviewed 7 cases of air embolism with 5 fatalities [15]. We had 3 cases of air embolism. All 3 patients survived without any residual damage because of early recognition and correct management by vigilant anaesthesiologists. In 2 cases, this problem occurred during resection of myoma and endometrium. However, in the third case embolism occurred during diagnostic hysteroscopy after a difficult cervical dilation. The sequence of events in all these cases were fall in end tidal $\mathrm{CO}_{2}$, followed by hypoxia, tachycardia, hypotension, cardiovascular collapse and subsequent asystole. Immediately hysteroscope was removed, patient turned to the left side in order to keep the gas on right side and thus decreasing the chance of paradoxical embolisation. A precordial thump was given to break the air pocket. Cardiopulmonary resuscitation was started and simultaneously central venous catheter was introduced and gas aspirated from right side of heart.

Various preventive measures recommended are :-

1. Avoid Trendelenburg position as it places the uterus above the level of heart and creates a venous vacuum with each diastolic relaxation.

2. Minimize cervical trauma and if required, use osmotic dilators preoperatively.

3. Always keep the os occluded so as to prevent entry of room air. Keep the last dilator inside till resectoscope is assembled. Whenever electrode is to be changed keep the obturator inside.
4. Always keep anaesthesiologists informed about the operative procedures like TCRE and myoma resection which can open venous sinuses and thus potential portals of air entry. Anaesthesiologists during such procedures can closely monitor end tidal $\mathrm{CO}_{2}$ (expired $\mathrm{CO}_{2}$ measurement of each breath amounts to non invasive estimation of $\mathrm{PaCO}_{2}$ ) and diagnose air embolism early. Doppler and transoesophageal echocardiography are not recommended for monitoring because of high false positive readings [15].

\section{Conclusion}

With proper preoperative evaluation, meticulous technique and vigilance for impending problems, complications of hysteroscopic surgery are largely preventable. Fluid overload is the commonest complication. Hysteroscopists must know the various types of media and peculiar problems associated with each so that he can make surgery safe. The knowledge of physiology and management of air embolism is mandatory for any active hysteroscopist. With ongoing research and advancement in technology hysteroscopic surgery is going to become more safe and less morbid.

\section{References}

1. Corfman RS, Diamond MP, DeCherney A. Complications of laparoscopy and hysteroscopy. Boston, Blackwell scientific publications 1993;177-86.

2. Loffer FD. The need to monitor intrauterine pressure-myth or necessity? J Am Assoc Gynecol Laproscopists 1994;2:1-2.

3. Rythen Alder E, Brundin J. Detection of carbon dioxide embolism during hysteroscopy. Gynecol Endoscopy 1992;1:207-10.

4. Golan A, Sieder M. High output left ventricular failure after Dextran use in an operative hysteroscopy. Fertil Steril 1990:54:939-41.

5. Leake JF, Murphy AA, Zacur HA. Noncardiogenic pulmonary oedema, complication of operative hysteroscopy. Fertil Steri] 1987:48:497-9.

6. Jedeikin R, Olsfanger D. Kessler I. Disseminated intravascular coagulopathy and adult respiratory distress syndrome. Life threatening complications of hysteroscopy. Am J Obstet Gynecol, 1990:162:44-5.

7. Ellingson TL, Aboulafia DM. Dextran syndrome, acute hypotension, noncardiogenic pulmonary oedema, anaemia and coagulopathy following hysteroscopic surgery using $32 \%$ Dextran 70. Chest, 1997;111:513-8.

8. Witz CA. Complications associated with absorption of hysteroscopic fluid media. Fertil Steril. 1993;60:745.

9. Ayus JC. Wheeler JM, Arieff Al. Postoperative hyponatraemic encephalopathy in menstruant women. Ann Intern Med, 1992;117:891-7.

10. Philips DR, Milim SJ, Nathanson HG, et al. Preventing hyponatremic encephalopathy : comparison of serum sodium and osmolality during operative hysteroscopy with $5 \%$ mannitol and $1.5 \%$ glycine as distension media. J Am Assoc 
Gynecol Laprosc 1996:4567-76.

11. Isaacson KB, Olive DL. Operative hysteroscopy in physiologic distension media. J Am Assoc Gynecol Laprosc 1999;6:1 13-8.

12. MacDonald R, Phipps J, Singer A. Endometrial ablation, a safe procedure. Gynaecological Endoscopy 1992;1:7-9.

13. Goldrath MJ. Uterine tamponade for control of acute uterine bleeding. Am J Obstet Gynecol, 1983;147:869-72.

14. Kivnick S, Kante MK. Bowel injury from roller ball ablation of endometrium. Obstet Gynecol, 1992;79:833.

15. Brooks PG. Venous air embolism during operative hysteroscopy. J Am Assoc Gynecol Laparosc 1997;4:399-402. 